\title{
A systematic review of hospital accreditation: the challenges of measuring complex intervention effects
}

\author{
Kirsten Brubakk ${ }^{*}$, Gunn E. Vist ${ }^{2}$, Geir Bukholm${ }^{3}$ Paul Barach ${ }^{4}$ and Ole Tjomsland ${ }^{5}$
}

\begin{abstract}
Background: The increased international focus on improving patient outcomes, safety and quality of care has led stakeholders, policy makers and healthcare provider organizations to adopt standardized processes for evaluating healthcare organizations. Accreditation and certification have been proposed as interventions to support patient safety and high quality healthcare. Guidelines recommend accreditation but are cautious about the evidence, judged as inconclusive. The push for accreditation continues despite sparse evidence to support its efficiency or effectiveness.

Methods: We searched MEDLINE, EMBASE and The Cochrane Library using Medical Subject Headings (MeSH) indexes and keyword searches in any language. Studies were assessed using the Cochrane Risk of Bias Tool and AMSTAR framework. 915 abstracts were screened and 20 papers were reviewed in full in January 2013. Inclusion criteria included studies addressing the effect of hospital accreditation and certification using systematic reviews, randomized controlled trials, observational studies with a control group, or interrupted time series. Outcomes included both clinical outcomes and process measures. An updated literature search in July 2014 identified no new studies.
\end{abstract}

Results: The literature review uncovered three systematic reviews and one randomized controlled trial. The lone study assessed the effects of accreditation on hospital outcomes and reported inconsistent results. Excluded studies were reviewed and their findings summarized.

Conclusion: Accreditation continues to grow internationally but due to scant evidence, no conclusions could be reached to support its effectiveness. Our review did not find evidence to support accreditation and certification of hospitals being linked to measurable changes in quality of care as measured by quality metrics and standards. Most studies did not report intervention context, implementation, or cost. This might reflect the challenges in assessing complex, heterogeneous interventions such as accreditation and certification. It is also may be magnified by the impact of how accreditation is managed and executed, and the varied financial and organizational healthcare constraints. The strategies hospitals should impelment to improve patient safety and organizational outcomes related to accreditation and certification components remains unclear.

Keywords: Accreditation, Certification, Hospital, Patient Safety, Evaluation

\footnotetext{
* Correspondence: kirsten.brubakk@helse-sorost.no

${ }^{1}$ South-Eastern Norway Regional Health Authority, Hamar, Norway

Full list of author information is available at the end of the article
} 


\section{Background}

Patient safety and patient centered care are emerging as key drivers in healthcare reform.

Accreditation is the most frequently external quality assessment of healthcare organizations' strategic goals [1]. We defined hospital accreditation programs as the systematic assessment of hospitals against accepted standards [2] and certification is a confirmation of characteristics of an object, person, or organization against published standards [3]. Little information is available on effective accreditation and certification strategies. Prominent national organizations have recommended accreditation which is being implemented widely. However, little evidence supports their effect on patient outcomes or other important markers such as core measures, organizational culture nor reliability.

Hospital accreditation was started by The American College of Surgeons 100 years ago, and since then the number of hospital accreditation programs has expanded rapidly. The World Health Organization identified 36 nationwide healthcare accreditation programs in 2000 [4]. Accreditation is an essential part of healthcare systems in more than 70 countries and is often provided by external and independent review, assessment or audit [5]. The systematic evaluation of healthcare services is a way to obtain regulatory peer review on the organizational maturity and reliability [6]. Literature reviews on the effects of accreditation on the quality of care do not provide strong evidence due to limitations of the studies [7-12].

Greenfield and Braithwaite [7] identified the effects of accreditation on promoting change and professional development, indicating that the effects were probably due to accreditation and certification, but lacking firm evidence. A systematic review by Nicklin et al. [8] found several positive benefits of accreditation, however, the study lacked rigor to support their conclusions. Shaw et al. [13] found evidence for positive effects between accreditation, certification and clinical leadership, systems for patient safety and clinical review, but was fell short of endorsing accreditation, and concluded with recommending further analysis to explore the association of accreditation and certification with clinical outcomes. Furthermore, Ho et al. have demonstrated an unintended negative impact on the learning environment of medical students and trainees, including decreased clinical learning opportunities, increased non-clinical workload, and violation of professional integrity in preparation and during accreditation and certification [14].

The aim of this study is to systematically assess the effects of accreditation and/or certification of hospitals on both organizational processes and outcomes.

\section{Methods}

We searched for published articles that assessed the effects of accreditation and/or certification of hospitals. The studies were reviewed for their research design and internal validity. We assessed each study's findings in regard to their effects on patient mortality, morbidity, patient safety, as well as process outcomes.

\section{Data sources and search strategy}

We searched MEDLINE, EMBASE, CRD, and the Cochrane Library, including the Cochrane Database of Systematic Reviews (CDSR), Database of Abstracts of Reviews of Effects (DARE) and Health Technology Assessment Database (HTA) for all studies on accreditation/certification in 2006 [11], and this was repeated in 2009 [12], 2013 and 2014. The same search criteria were used to monitor the studies addressing effect of accreditation/certification in hospitals.

The search was designed and conducted by an information specialist librarian who updated the search strategies from 2006 to 2009 and used the combinations of key words and Medical Subject Heading terms (MeSH) related to accreditation, certification and hospitals. The reference lists of selected articles were searched for potentially relevant studies meeting the inclusion criteria (snowballing). In addition, we used Google search engine using the search words accreditation, certification and patient safety. We updated our literature search in July 2014, searching the same databases with the same inclusion criteria. We found no relevant additional studies to include in our analysis.

\section{Study selection}

We included studies identified in any language using the search strategy with the following study design: systematic reviews, randomized controlled trials (RCT), nonrandomized controlled trials, controlled before and after studies (CBAs), and interrupted time series (ITS) (defined as at least three measurements before and three after the introduction of accreditation and/or certification).

The inclusion criteria used were:

Population: all types of hospitals were included. Intervention: all types of accreditation and/or certification of hospitals.

Comparison: any hospital that was not accredited or certified, either by not seeking or not receiving accreditation and/or certification.

Outcomes: both clinical outcomes and process measures.

Two of the authors (GEV, KB) independently reviewed all titles, references and abstracts generated by the original search in order to identify articles for potential 
inclusion. All reports, independent of language, were evaluated for the inclusion criteria.

Each article considered potentially eligible according to the chosen criteria was independently read in full text and then assessed using a standardized form for internal validity by two authors (GEV, KB,). If several estimates for one study outcome were reported, the most fully adjusted estimate was abstracted. Each assessment was conducted independently by two reviewers, the results were compared, and the differences were all reconciled by consensus.

This study did not involve human material or human data, so an ethic approval was not needed. No written consent was obtained from participants for this literature study. Additional file 1 provides a complete description of the search strategies; and Additional file 2 provides a detailed overview of the updated search results. The PRISMA checklist (Preferred Items for Systematic Reviews and Meta-Analyses) was used for this systematic review. Please see Additional file 3.

\section{Results \\ Search results}

Our search of electronic databases identified a considerable increase in studies addressing the effect of accreditation and/or certification. In 2006, 672 studies were identified [11]. Over the next 3 years 522 new studies were published. In 2013 we identified another 910 relevant studies. Of the identified studies in 2013 fifteen citations were considered potentially eligible based on the inclusion criteria. Two additional articles were identified, and an additional three references were identified by manually searching the articles' reference lists. Twenty references were considered potentially eligible and were retrieved for a full text assessment. Of these, 16 articles were excluded because they did not fulfil the inclusion criteria; Table 4 presents the excluded studies and the detailed reasons for their exclusion.

The agreement between reviewers for study eligibility was complete. As only one original study was included a meta-analysis was not possible (Fig. 1).

\section{Characteristics of included studies}

We included systematic reviews as well as controlled studies. A total of four references, three systematic reviews and one primary study met the inclusion [15-18]. The aims and the inclusion criteria of the three reviews were slightly different. However, their inclusion criteria overlapped with the inclusion criteria for this review. Please see Table 1 for included systematic reviews in this review.

The qualities of the systematic reviews were assessed using the AMSTAR quality checklist framework, the standard for assessing methodological quality of systematic reviews [19]. The results of the assessment are shown in Table 2 . Two of the reviews were of moderate quality scoring
6/11 [17], and 7/11 [16], respectively, whereas the third review was scored as high quality with a score of 9/11 [15]. Our review scored 9/11 in the AMSTAR assessment. The primary study [18] was assessed as having a high risk of bias after using the risk of bias assessment as described in the Cochrane Handbook for randomized controlled trails [20]. The assessment is shown in Table 3.

\section{Included systematic reviews}

The Cochrane review by Flodgren et al. has the best quality AMSTAR score [15]. The authors identified two studies which met their inclusion criteria that focused on the effect of external inspection on: a) compliance with standards improving healthcare organizations; b) healthcare professional behavior; and, c) patient outcomes.

The first study was a cluster-randomized controlled trial by Salmon et al. [18] that involved 20 South African public hospitals. The other study was an interrupted time- series conducted to identify the effects of the NHS Healthcare Commissions Infection Inspection program on the MRSA rates in UK trusts hospitals, but did not meet our inclusion criteria. Flodgren et al. concluded that the results could not be used to draw firm conclusions on the effectiveness of external inspection.

The Matrix Knowledge group searched the literature in 2010 and found 56 articles that addressed the impact of hospital accreditation [16]. The vast majority of these studies used surveys with standardized questionnaires, and reported staff, patient and stakeholders' perceptions of impact. Overall they reported a positive impact of accreditation on hospital and professional practice. Only the South African cluster-randomized controlled trial was consistent with the inclusion criteria of our study.

Alkhenizan and Shaw searched the literature in 2009 and included 26 studies that assessed either the general impact of accreditation on hospitals or impact on a single aspect of performance of healthcare services, and on subspecialty accreditation programs. The authors found a positive effect of accreditation on improving the process of care and clinical outcomes [17]. Sixteen (62\%) of the 26 included studies reported significant positive results attributed to accreditation, mainly related to better compliance with guidelines. Ten studies $(38 \%)$ reported weak or no improvement after accreditation. Alkhenizan and Shaw included the one RCT by Salmon et al. [18].

\section{Included primary study}

There was one primary study identified that met all of our criteria, the randomized controlled trial from South Africa by Salmon et al. [18]. This study was not identified through the database search, but by searching reference lists (snowballing); it was missed by our literature review in 2009. The authors included 20 hospitals in their study. The hospitals were randomly selected and 
915 Titles/abstracts reviewed. 910 Articles from PubMed, Embase, Cochrane. 5 Articles from reference lists

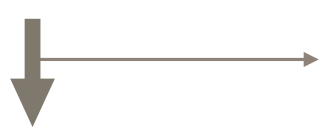

20 Potentially relevant articles retrieved for full-text review.

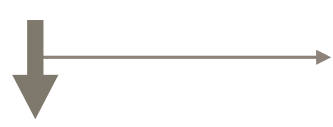

4 Articles included. Three review articls and 1 randomized controlled study abstracted.
895 Excluded based on review of title and abstract.

\section{Excluded,based on full-} text assessment.

Fig. 1 Flowchart. Flowchart of study selection process. Database searched January 18, 2013

stratified into groups according to hospital size (number of beds). Ten hospitals were randomized to start an accreditation program, while the other 10 served as controls. Two sets of data, before and after measures, were collected by the Council for Health Services Accreditation of Southern Africa (COHSASA), and by independent research teams. Initially, 12 indicators of hospital care quality were identified and used for the first data collection - this number was reduced to eight in the second data collection. Of these indicators, surgical wound infection, time to surgery, neonatal mortality rate and financial solvency were left out due to challenges in data collection. It is unclear whether the four indicators that were abandoned would have influenced the overall magnitude, range of results or conclusions of the study.

The compliance with the COHSASA accreditation standard was found to have increased substantially in the accredited hospitals $(p<0.001)$, whereas the control hospitals maintained their score throughout the study. Eight hospital quality indicators were reported. The nurses' perceptions of clinical quality was increased in the accredited hospitals $(p=0.031)$; however, the other seven indicators showed little or no effect on the quality indicators; patient satisfaction with care $(p=0.484)$; patient medication education $(p=0.395)$; accessibility of medical records $(p=0.492)$; completeness of medical records $(p=0.114)$; completeness of peri-operative notes $(p=0.489)$; labelling of ward stock $(p=0.112)$; and, composite assessment of hospital sanitation $(p=0.641)$.

\section{Excluded studies}

Sixteen of the 20 studies were excluded after they were independently evaluated by two researchers (GVE, KB). The reasons for exclusion were as follows: four studies had no control groups [21-24]; two performed the study outside hospitals $[25,26]$; four studies did not report on the effects of accreditation [27-30]; two studies lacked baseline measurements [31, 32]; one study lacked description of the accreditation intervention [33]; two studies did a comparison of the clinical outcome in accredited hospitals with outcome in non-accredited hospitals, but did not assess the effect of the intervention per se. [34, 35]; and, one systematic review conducted a qualitative assessment of healthcare professionals' attitude toward accreditation, but the effect of the intervention was not assessed [36]. A complete list of the excluded studies and the reasons for their exclusion is presented in Table 4. 
Table 1 Included systematic reviews

\begin{tabular}{|c|c|c|c|c|c|c|}
\hline Reference & Search date & Aim of review & Study design included & Number of included studies & Main conclusion stated by authors & $\begin{array}{l}\text { Studies that match } \\
\text { our inclusion criteria }\end{array}$ \\
\hline $\begin{array}{l}\text { Flodgren et al. } \\
2011[15]\end{array}$ & May 2011 & $\begin{array}{l}\text { Evaluate the effectiveness of external } \\
\text { inspection of compliance with } \\
\text { standards in improving healthcare } \\
\text { organizations behavior, healthcare } \\
\text { professionals behavior and patient } \\
\text { outcomes }\end{array}$ & $\mathrm{RCT}, \mathrm{CCT}, \mathrm{ITS}, \mathrm{CBA}$ & Two in total, 1 RCT, 1 ITS & $\begin{array}{l}\text { No firm conclusion were drawn } \\
\text { due to paucity of high-quality } \\
\text { controlled evaluations }\end{array}$ & Salmon et al. [18] \\
\hline $\begin{array}{l}\text { Matrix Knowledge } \\
\text { group } 2010 \text { [16] }\end{array}$ & August 2010 & $\begin{array}{l}\text { Produce a general overview of results } \\
\text { obtained and methodologies used } \\
\text { to assess impact of accreditation }\end{array}$ & $\begin{array}{l}\text { Studies containing an } \\
\text { element of comparison }\end{array}$ & $\begin{array}{l}56 \text { in total, } 40 \text { studies with } \\
\text { quantitative design of which } \\
1 \text { study presented empirical } \\
\text { data }\end{array}$ & $\begin{array}{l}\text { Most studies suggest that } \\
\text { accreditation/certification has an } \\
\text { impact on the organization or on } \\
\text { the professional practice. The impact } \\
\text { on health outcomes or improvement } \\
\text { in these outcomes was not } \\
\text { demonstrated. }\end{array}$ & Salmon et al. [18] \\
\hline $\begin{array}{l}\text { Alkhenizan \& Shaw } \\
2011 \text { [17] }\end{array}$ & June 2009 & $\begin{array}{l}\text { Evaluate the impact of accreditation } \\
\text { programs on the quality of healthcare } \\
\text { services }\end{array}$ & $\begin{array}{l}\text { Clinical trials, observational } \\
\text { studies and qualitative studies }\end{array}$ & $\begin{array}{l}26 \text { in total, } 10 \text { studied } \\
\text { accreditation of hospitals of } \\
\text { which } 1 \text { had a hospital control } \\
\text { group }\end{array}$ & $\begin{array}{l}\text { Accreditation improves the process } \\
\text { of care provided by healthcare } \\
\text { services }\end{array}$ & Salmon et al. [18] \\
\hline
\end{tabular}


Table 2 AMSTAR, assessing methodological quality of systematic reviews

\begin{tabular}{|c|c|c|c|c|}
\hline Study & $\begin{array}{l}\text { Alkhenzian et al. } \\
2011\end{array}$ & $\begin{array}{l}\text { Matrix group } \\
2010\end{array}$ & $\begin{array}{l}\text { Flodgren et al. } \\
2011\end{array}$ & Brubakk et al. \\
\hline AMSTAR question & \multicolumn{4}{|c|}{ Yes, No, Can't answer, Not applicable } \\
\hline 1. Was an 'a priori' design provided? & Yes & Yes & Yes & Yes \\
\hline 2. Was there duplicate study selection and data extraction? & No & No & Yes & Yes \\
\hline 3. Was a comprehensive literature search performed? & Yes & Yes & Yes & Yes \\
\hline $\begin{array}{l}\text { 4. Was the status of publication (i.e. grey literature) used } \\
\text { in the inclusion criterion? }\end{array}$ & No & Yes & No & No \\
\hline 5. Was a list of studies (included and excluded) provided? & $\begin{array}{l}\text { Yes, although only } \\
\text { for the included }\end{array}$ & $\begin{array}{l}\text { Yes, although only } \\
\text { for the included }\end{array}$ & $\begin{array}{l}\text { Yes, both included } \\
\text { and excluded studies }\end{array}$ & $\begin{array}{l}\text { Yes, both included } \\
\text { and excluded studies }\end{array}$ \\
\hline 6. Were the characteristics of the included studies provided? & Yes & Yes & Yes & Yes \\
\hline $\begin{array}{l}\text { 7. Was the scientific quality of the included studies assessed } \\
\text { and documented? }\end{array}$ & Yes & Yes, No & Yes & Yes \\
\hline $\begin{array}{l}\text { 8. Was the scientific quality of the included studies used } \\
\text { appropriately in formulating conclusions? }\end{array}$ & No & Can't answer & Yes & Yes \\
\hline $\begin{array}{l}\text { 9. Were the methods used to combine the findings of } \\
\text { studies appropriate? }\end{array}$ & Not applicable (N/A) & Yes & Not applicable (N/A) & Not applicable (N/A) \\
\hline 10. Was the likelihood of publication bias assessed? & No & No & Yes & Yes \\
\hline 11. Was the conflict of interest stated? & No & No & Yes & Yes \\
\hline
\end{tabular}

AMSTAR. Assessing Methodological Quality of Systematic Review, quality assessment of included systematic reviews categorized by yes, no, cannot answer, not applicable

\section{Narrative review of excluded studies}

We identified one primary study and three systematic reviews. Notably, we had strict inclusion criteria and found few studies that met these strict criteria. A summary of the methods used in the excluded studies is relevant to the discussion on assessing the full measure of complex interventions. Accreditation was addressed in several ways in the publications that failed to fulfil the criteria for inclusion in the present review. Seven of the 16 excluded studies conducted cross-sectional studies

Table 3 Risk of bias assessment of study by Salmon et al. [18]

\begin{tabular}{|c|c|c|}
\hline Domain & Support for judgement & Review author's judgement \\
\hline \multicolumn{3}{|l|}{ Selection bias } \\
\hline Random sequence generation & $\begin{array}{l}\text { They state stratified randomisation, but give no } \\
\text { information about the procedure }\end{array}$ & Unclear \\
\hline Allocation concealment & Not mentioned & Unclear \\
\hline \multicolumn{3}{|l|}{ Performance bias } \\
\hline Blinding of participants and personnel & $\begin{array}{l}\text { Not mentioned and appears impossible/not } \\
\text { possible to blind hospitals }\end{array}$ & Unclear \\
\hline \multicolumn{3}{|l|}{ Detection bias } \\
\hline Blinding of outcome assessor & Not mentioned & Unclear \\
\hline \multicolumn{3}{|l|}{ Attrition bias } \\
\hline Incomplete outcome date & $\begin{array}{l}\text { The largest hospital did not complete the study. } \\
\text { Follow- up time was shortened because controls } \\
\text { wanted to receive the intervention }\end{array}$ & High risk \\
\hline \multicolumn{3}{|l|}{ Reporting bias } \\
\hline Selective reporting & $\begin{array}{l}\text { Outcome selection conducted by participants and } \\
\text { accreditor. Many outcomes/ indicators were } \\
\text { dropped from the follow- up measurement }\end{array}$ & High risk \\
\hline \multicolumn{3}{|l|}{ Other bias } \\
\hline Other sources of bias & $\begin{array}{l}\text { This was a cluster randomized trial, adjustment for } \\
\text { clustering in analysis of results were not mentioned }\end{array}$ & Unclear \\
\hline
\end{tabular}

${ }^{\mathrm{a}}$ The risk of bias assessment as described in the Cochrane Handbook for randomized controlled trails [20]

Risk of bias assessment of the included primary study by Salmon el at [18]

SOURCE: Higgins J, Green S. Cochrane Handbook for Systematic Reviews of Interventions Version 5.1.0 [updated March 2011]. The Cochrane Collaboration, 2011 
Table 4 Excluded studies

\begin{tabular}{lll}
\hline Reference (country) & Reason cited for exclusion & Aim of study \\
\hline Al Awa et al. 2011 [22], Saudi Arabia & No control group & $\begin{array}{l}\text { Determine if patient safety and quality care } \\
\text { indicators improve post accreditation }\end{array}$ \\
Al Awa et al. 2011 [23], Saudi Arabia & No control group & $\begin{array}{l}\text { Evaluate nursing perception of care/safety } \\
\text { after accreditation }\end{array}$ \\
Al Tehewy et al. 2009 [26], Egypt & Not in hospital (health units) & $\begin{array}{l}\text { Determine the effects of accreditation of } \\
\text { non-governmental organizations }\end{array}$ \\
Chen et al. 2003 [31], USA & Measured outcome at only one point & $\begin{array}{l}\text { Identify association between JCAHO } \\
\text { accreditation and quality of care for acute } \\
\text { myocardial infection }\end{array}$ \\
Chuang et al. 2009 [27], Australia & Did not measure effect & $\begin{array}{l}\text { Propose an integrated research model } \\
\text { du Bois et al. } 2009 \text { [33], Germany }\end{array}$ \\
& Review not linked to accreditation & $\begin{array}{l}\text { Evaluate the impact of different physician } \\
\text { and hospital characteristics on outcome } \\
\text { in ovarian cancer patients }\end{array}$
\end{tabular}

Gokenbach et al. 2011, USA [24] No control group

Lichtman et al. 2009 [28], USA

Lichtman et al. 2011 [32], USA

Menachemi et al. 2008 [25], USA

V Phua et al. 2011 [29], Singapore/Asia

Al-Awa et al. 2012 [30], Saudi Arabia

Alkenizan \& Shaw 2012 [36], UK,

Bohmer et al. 2012 [21], Germany

Schmaltz et al. 2011 [34], USA

Nguyen et al. 2012 [35], USA of accreditation
Did not measure effects of accreditation

Measured outcome at only one point

Not in hospital

Did not evaluate effects of accreditation

Compares survey results in accredited hospital to international results

Review, Qualitative assessment of attitude, did not measure effect

No controls

Compared the difference in development of accredited vs. non accredited hospitals, not the effects

Compared the difference in development of accredited vs. non accredited hospitals, not the effects of accreditation
Identify the effects of Magnet accreditation on one hospital

Determine whether hospitals certified had better outcome within the first year of accreditation than non accredited hospitals

Identify outcomes after ischemic stroke for hospitals with and without TJC certification

Identify quality outcome in accredited and non-accredited ambulatory surgical centers

Assess compliance to sepsis bundles in intensive care units in Asia

Perform an unbiased assessment of the impact of accreditation on patient safety culture

Review the literature of the attitude of healthcare professionals towards accreditation

Identify to which extent pain management standards was implemented in hospitals after accreditation

Examine the association between Joint Commission accreditation status and both absolute measures and trends in hospital performance

Analyze the peri-operative outcomes of bariatric surgery performed at accredited vs. non accredited centres

Excluded studies after full text assessment presenting aim of study and reason for exclusion

comparing patient outcome in accredited and nonaccredited hospitals [23, 25, 31, 32, 34, 35]. In general, performance in accredited hospitals was higher than in non-accredited hospitals and showing higher compliance to standards also affecting outcome positively [29]. The study by Lichtman et al. identified the risk of selection bias as certified hospitals had better outcome than non-certified hospitals even before the program began [28]. Four studies used survey to assess the staff and patient' perception of patient safety culture, quality and patient satisfaction pre- and post accreditation $[21,22,26,30]$. Nurses and patients reported that positive changes in their organization were a result of accreditation, while physicians in general were more sceptical. This is consistent with the findings in Alkhenizan and Shaw's systematic review studying healthcare professionals' attitude toward accreditation. Nurses in general were more favourably inclined than physicians indicating the necessity of special education schemes to involve staff in the accreditation process [36]. Another systematic review did not address accreditation directly, but found that physician specialization had effect on outcome of ovarian cancer patients [34]. A study on implementing nurse accreditation in one hospital reports increased staff and 
patient satisfaction, improved nurse-physician relationship, improved nursing quality and reduced turnover and vacancy rates [24]. The last study aims at proposing an integrated research model of the accreditation and quality measurement/reporting systems, providing more supportive information on the system weakness [27].

\section{Discussion}

In this systematic review, we examined 20 studies involving accreditation and certification aimed at improving patient and organizational outcomes. Because few studies specifically addressed the correlation between accreditation and certification of hospitals and patient outcomes, we could not reach firm conclusions regarding effective strategies in this area.

This is no surprise as accreditation is anticipated as a prototypical example of a complex intervention. Within our classification of interventions, the manner in which the studies carried out specific interventions varied widely. There is complexity in the intervention components as well as in the theoretical background of the intervention, the implementation context, and the targeted outcomes [37]. The literature is dominated by descriptive studies attributing changes in the organization to the accreditation process. The research has ranged from identifying the change in compliance to standards, patient satisfaction, performance indicators, health professionals' satisfaction and an overall review of the perceptions of accreditation and/or certification among patients, professionals and other stakeholders. Many of the studies we reviewed were heterogeneous, uncontrolled and fraught with confounding variables, adding little clarity or guidance. Despite the lack of convincing evidence there is no reason to believe that accreditation and certification will be abandoned. The lack of documented effect may simply mean that due to the heterogeneity of study design and methods much uncertainty remains regarding its putative effects.

The paucity of evidence is highlighted by our systematic search that revealed variable degrees of rigor. The search identified only one controlled study, the randomized trial from South Africa from 2003. The study, however, is weak scientifically, and does not address morbidity or patient safety measures well enough to support any conclusions across a wide range of safety systems examined.

The methodological challenges of measuring the effects of accreditation/certification are increased by the complexity of the hospital organizations and their heterogeneous components. It is unclear what elements are being subjected to assessment [38, 39]. The UK Medical Research Council points out that it is hard to identify the "active ingredient" of complex interventions such as falls prevention or hand washing campaigns, as these interventions comprise many separate, multi-level and concurrent elements [40]. Furthermore, the interventions are interpreted in many ways and are used in different settings which strongly complicate the evaluation of the effects $[41,42]$. Lessons can be learned from noncontrolled studies such as cross-sectional studies. Comparison between accredited and non-accredited hospitals yields important information about potential differences between these hospitals, but cannot provide information about the observed variations, and whether the results are transferable to other settings.

It is noteworthy that there was a low level of methodological rigor in most of the studies included in this review, as outcome measures were ambiguous and only limited operational details were reported. Significant methodological challenges such as self-selection and lack of robust controls undermine the ability to extrapolate or infer from the published literature if these effects were caused by accreditation and/or certification [43]. Even though our systematic review was conducted carefully adhering to the Cochrane guidelines, we were unable to find conclusive evidence on accreditation and certification. Some studies surveyed staff, stakeholders or other hospital representatives before, during and after a certification and/or accreditation process. Some studies show higher quality in accredited hospitals when compared to non-accredited hospitals, but it is uncertain if this is the result of accreditation, self-selection or is due to other extraneous factors.

Working with predetermined inclusion criteria allowed a specialized literature search which generally increases the chances of finding all relevant studies although it only identifies the published literature. Reports of studies that are only posted on the web pages of organizations or stakeholders (grey literature) are more difficult to find. Notably, the randomized controlled trial from South Africa was only available as grey literature and was not identified through a systematic literature search of electronic databases. Although unlikely, it is possible that there may be other studies that the present or other reviews have missed.

Our study has several limitations. An unavoidable limitation of systematic reviews is that they may appear out-dated rapidly as new studies are published; hence, our review only included recently published systematic reviews. Notably, we repeated the search in July 2014 to ensure that we captured any new studies. Future investigations might control for case mix and time trends, employ suitable comparison groups, and consider other analytic approaches for analyzing time series data such as interrupted time series data, or ARIMA methods [44]. Interrupted time series analyses, Bayesian analysis and ARIMA may be suited for adjusting for clustering of effects within sites, while accounting for patient-level 
effects, and site-level structural measures. Studies addressing how and why the interventions might work, rather than just the effects of the intervention, might provide valuable information on complex interventions [39].

\section{Conclusions}

Hospitals are now faced with the challenge of improving their patient outcomes and reliability. Our study provides a comprehensive overview of the effects of accreditation and/or certification of hospitals on quality and patient safety outcomes and concludes that due to scant evidence, no conclusions could be reached to support its effectiveness. The accreditation programs require substantial financial and labor investments, and distract healthcare teams from their primary clinical goals. Accordingly further research on the clinical impact of these programs is needed, and it is important to weigh the transactional opportunity and financial costs of accreditation against other financial investments in quality improvement interventions [45-48]. Furthermore, we found little guidance demonstrating the cost effectiveness of accreditation and/or certification.

In summary, we found that the proven role of accreditation and certification in improving patient and organizational outcomes remain largely undefined. Accreditation and certification is a thriving industry and there are many interested stakeholders who may profit on promoting these services despite the lack of robust evidence of their effectiveness $[49,50]$. Finally, because hospitals are expending resources on accreditation and/ or certification they may not be able to address other, more pressing patient safety issues [51]. There is little reason to believe however that accreditation or certification will be abandoned because of the lack of empirical evidence of its effects, so future contributions should probably focus on what aspects of accreditation serve a useful purpose, rather than focusing on "does it work".

Before planning further studies to evaluate impact of accreditation and certification efforts, a more thorough and nuanced analysis of the available evidence about which components of accreditation/certification seem to be most effective in enabling patient centered, high quality and safer outcomes should be performed [37].

\section{Additional files}

Additional file 1: Complete search strategy January 2013. Complete search strategy performed in PubMed (from 1948), EMBASE (from 1980), CRD, and the Cochrane Library, including the Cochrane Database of Systematic Reviews (CDSR), Database of Abstracts of Reviews of Effects (DARE) and Health Technology Assessment Database (HTA) January 2013.

Additional file 2: Complete search strategy July 2014. Complete search strategy performed in PubMed (from 1948), EMBASE (from 1980), CRD, and the Cochrane Library, including the Cochrane Database of
Systematic Reviews (CDSR), Database of Abstracts of Reviews of Effects (DARE) and Health Technology Assessment Database (HTA) July 2014.

Additional file 3: PRISMA 2009 Checklist. PRISMA 2009 Checklist.

Transparent reporting of systematic reviews and meta-analyses. Prisma-statement.org.

\section{Competing interests}

The authors declare that they have no competing interests.

\section{Authors' contributions}

GEV and KB designed and managed the study. GEV and KB reviewed title, references and abstracts. All authors (GEV, KB, GB, PB, and OT) were involved in the interpretation of the data. KB, PB and OT drafted the manuscript. All authors read and approved the final version.

\section{Authors' information}

${ }^{1}$ KB: RN, MHA, South-Eastern Norway Regional Health Authority, Norway. GEV: PhD, Norwegian Knowledge Centre for the Healthcare Services, Norway. ${ }^{3}$ GB: MD, PhD, MHA, MPH, Norwegian Institute of Public Health and Norwegian University of Life Science, Norway. ${ }^{4}$ PB: MD, MPH, Wayne State University School of Medicine, US. ${ }^{5}$ OT: MD, PhD, South-Eastern Norway Regional Health Authority, Norway.

\section{Acknowledgement}

All persons contributing to the manuscript met the criteria of authorship. We did not use a language editor or a scientific writer. There was no external funding for this paper.

\section{Author details}

${ }^{1}$ South-Eastern Norway Regional Health Authority, Hamar, Norway. ${ }^{2}$ Prevention, Health promotion and Organization Unit, Norwegian Knowledge Centre for the Healthcare Services, Oslo, Norway. ${ }^{3}$ Norwegian Institute of Public Health, Oslo, Norway. ${ }^{4}$ Wayne State University School of Medicine, Michigan, USA. ${ }^{5}$ Department of Medicine and Health,

South-Eastern Norway Regional Health Authority, Hamar, Norway.

Received: 12 March 2015 Accepted: 29 June 2015

Published online: 23 July 2015

\section{References}

1. Viswanathan HN, Salmon JW. Accrediting organizations and quality improvement. Am J Manage Care. 2000;6(10):1117-30.

2. Jovanovic B. Hospital accreditation as method for assessing quality in health care. Arch Oncol. 2005;13(3/4):156.

3. Shaw C, Groene O, Mora N, Sunol R. Accreditation and ISO certification: do they explain differences in quality management in European hospitals? Int J Qual Health Care. 2010;22(6):445-51.

4. Shaw CD. Evaluating accreditation. Int J Qual Health Care. 2003;15(6):455-6.

5. Greenfield D, Braithwaite J. Developing the evidence base for accreditation of healthcare organisations: a call for transparency and innovation. Qual Saf Health Care. 2009;18(3):162-3.

6. Grepperud S. Is the hospital decision to seek accreditation an effective one? Int J Health Plann Manage. 2015;30(1):E56-68.

7. Greenfield D, Braithwaite J. Health sector accreditation research: a systematic review. Int J Qual Health Care. 2008;20(3):172-83.

8. Nicklin W, Dickson S. The Value and Impact of Health Care Accreditation: A Literature Review. 2011.

9. Al-Awa B, De Wever A, Melot C, Devreux I. An overview of patient safety and accreditation: a literature review study. Res J Med Sci. 2011;5:200-23.

10. Hinchcliff R, Greenfield D, Moldovan M, Westbrook Jl, Pawsey M, Mumford $V$, et al. Narrative synthesis of health service accreditation literature. BMJ Qual Saf. 2012;21(12):979-91.

11. Vist GE, Nøstberg AM, Brubakk K, Munkeby BH. Effects of Certification and Accreditation of Hospitals. Oslo: Nasjonalt Kunnskapssenter for helsetjenester (Norwegian Knowledge Center for the Health Services); 2009. Report No.: 1890-1298/978-82-8121-315-9 Contract No.: Report.

12. Loland NW. Effekts av ISO sertifisering og akkreditering av sykehus (effects of ISO certification and accreditation in hospitals). Oslo: Nasjonalt Kunnskapssenter for helsetjenester (Norwegian Knowledge Center for the Health Services); 2006. Report No.: 82-8121-140-7 Contract No.: Report. 
13. Shaw CD, Groene O, Botje D, Sunol R, Kutryba B, Klazinga N et al. The effect of certification and accreditation on quality management in 4 clinical services in 73 European hospitals. Int J Qual Health Care. 2014. doi:10.1093/intqhc/mzu023.

14. Ho M-J, Chang H-H, Chiu Y-T, Norris JL. Effects of hospital accreditation on medical students: a national qualitative study in Taiwan. Acad Med. 2014:89(11):1533-9.

15. Flodgren G, Pomey MP, Taber SA, Eccles MP. Effectiveness of external inspection of compliance with standards in improving healthcare organisation behaviour, healthcare professional behaviour or patient outcomes. Cochrane Database Syst Rev. 2011;11:CD008992.

16. de Santé HA. Literature Review on the Impact of Hospital Accreditation. Paris: MATRIX Knowledge group; 2010.

17. Alkhenizan A, Shaw C. Impact of accreditation on the quality of healthcare services: a systematic review of the literature. Ann Saudi Med. 2011;31(4):407-16

18. Salmon JW, Heavens J, Lombard C, Tavrow P. The impact of accreditation on the quality of hospital care: KwaZulu-Natal Province, Republic of South Africa. Oper Res Results. 2003;2:17

19. Shea B, Grimshaw J, Wells G, Boers M, Andersson N, Hamel C, et al. Development of AMSTAR: a measurement tool to assess the methodological quality of systematic reviews. BMC Med Res Methodol. 2007;7(1):10

20. Higgins JPT, Altman DG. Assessing Risk of Bias in Included Studies. Cochrane Handbook for Systematic Reviews of Interventions. Chichester: Wiley; 2008. p. 187-241.

21. Bohmer AB, Poels M, Simanski C, Trojan S, Messer K, Wirtz MD, et al. Quality management in acute pain therapy: results from a survey of certified hospitals. Schmerz (Berlin, Germany). 2012;26(4):425-30, 32-4. doi:10.1007/ s00482-012-1205-X.

22. Al Awa B, Jacquery A, Almazrooa A, Habib H, Al-Noury K, El Deek B, et al. Comparison of patient safety and quality of care indicators between pre and post accreditation periods in King Abdulaziz University Hospital. Res J Med Sci. 2011;5(1):61-6.

23. Al Awa B, de Wever A, Almazrooa A, Habib H, al-Noury K, el Deek B, et al. The impact of accreditation on patient safety and quality of care indicators at King Abdulaziz University Hospital in Saudi Arabia. Res J Med Sci. 2011;5(1):43-51.

24. Gokenbach V, Drenkard K. The outcomes of magnet environments and nursing staff engagement: a case study. Nurs Clin North Am. 2011;46(1):89-105. doi:10.1016/j.cnur.2010.10.008.

25. Menachemi N, Chukmaitov A, Brown LS, Saunders C, Brooks RG. Quality of care in accredited and nonaccredited ambulatory surgical centers. Jt Comm J Qual Patient Saf. 2008;34(9):546-51.

26. Al Tehewy M, Salem B, Habil I, El Okda S. Evaluation of accreditation program in non-governmental organizations' health units in Egypt: short-term outcomes. Int J Qual Health Care. 2009;21(3):183-9.

27. Chuang S, Inder K. An effectiveness analysis of healthcare systems using a systems theoretic approach. BMC Health Serv Res. 2009;9:195.

28. Lichtman JH, Allen NB, Wang Y, Watanabe E, Jones SB, Goldstein LB. Stroke patient outcomes in US hospitals before the start of the Joint Commission Primary Stroke Center certification program. Stroke. 2009:40(11):3574-9.

29. Phua J, Koh Y, Du B, Tang YQ, Divatia JV, Tan CC, et al. Management of severe sepsis in patients admitted to Asian intensive care units: prospective cohort study. BMJ (Clinical research ed). 2011;342:d3245. doi:10.1136/bmj.d3245.

30. Al-Awa B, Al Mazrooa A, Rayes O, El Hati T, Devreux I, Al-Noury K, et al. Benchmarking the post-accreditation patient safety culture at King Abdulaziz University Hospital. Ann Saudi Med. 2012;32(2):143-50.

31. Chen J, Rathore SS, Radford MJ, Krumholz HM. JCAHO accreditation and quality of care for acute myocardial infarction. Health Aff. 2003;22(2):243-54.

32. Lichtman JH, Jones SB, Wang Y, Watanabe E, Leifheit-Limson E, Goldstein LB. Outcomes after ischemic stroke for hospitals with and without Joint Commission-certified primary stroke centers. Neurology. 2011;76(23):1976-82. doi:10.1212/WNL.0b013e31821e54f3.

33. du Bois A, Rochon J, Pfisterer J, Hoskins WJ. Variations in institutional infrastructure, physician specialization and experience, and outcome in ovarian cancer: a systematic review. Gynecol Oncol. 2009;112(2):422-36. doi:10.1016/j.ygyno.2008.09.036.

34. Schmaltz SP, Williams SC, Chassin MR, Loeb JM, Wachter RM. Hospital performance trends on national quality measures and the association with loint Commission accreditation. J Hosp Med. 2011;6(8):454-61. doi:10.1002/ jhm.905.
35. Nguyen NT, Nguyen B, Nguyen VQ, Ziogas A, Hohmann S, Stamos MJ. Outcomes of bariatric surgery performed at accredited vs nonaccredited centers. J Am Coll Surg. 2012;215(4):467-74. doi:10.1016/ j.jamcollsurg.2012.05.032.

36. Alkhenizan A, Shaw C. The attitude of health care professionals towards accreditation: a systematic review of the literature. J Family Community Med. 2012;19(2):74.

37. Köpke S, McCleery J. Systematic reviews of case management: too complex to manage? Cochrane Database Syst Rev. 2015;1:ED000096-ED.

38. Øvretveit J, Gustafson D. Evaluation of quality improvement programmes. Qual Saf Health Care. 2002;11(3):270-5. doi:10.1136/qhc.11.3.270.

39. Shepperd S, Lewin S, Straus S, Clarke M, Eccles MP, Fitzpatrick R, et al. Can we systematically review studies that evaluate complex interventions? PLoS Med. 2009;6(8):e1000086.

40. Council MR. A Framework for Development and Evaluation of RCTs for Complex Interventions to Improve Health. London: Medical Research Council; 2000.

41. Scrivens E. Assessing the value of accreditation systems. Eur J Public Health. 1997;7(1):4-8. doi:10.1093/eurpub/7.1.4.

42. Ovretveit J, Gustafson D. Using research to inform quality programmes. BMJ (Clinical research ed). 2003;326(7392):759-61. doi:10.1136/bmj.326.7392.759.

43. Lutfiyya MN, Sikka A, Mehta S, Lipsky MS. Comparison of US accredited and non-accredited rural critical access hospitals. Int J Qual Health Care. 2009;21(2):112-8.

44. Ramsay CR, Matowe L, Grilli R, Grimshaw JM, Thomas RE. Interrupted time series designs in health technology assessment: lessons from two systematic reviews of behavior change strategies. Int J Technol Assess Health Care. 2003;19(4):613-23.

45. Øvretveit J. Does improving quality save money. A review of evidence of which improvements to quality reduce costs to health service providers. London: The Health Foundation; 2009.

46. Shaw CD, Collins CD. Health service accreditation: report of a pilot programme for community hospitals. BMJ. 1995;310(6982):781.

47. Cross M. Money pit: is accreditation always worth the cost? Manag Care 2003;12(7):26-30.

48. Rockwell DA, Pelletier LR, Donnelly W. The cost of accreditation: one hospital's experience. Hosp Community Psychiatry. 1993;44(2):151-5.

49. Braithwaite J, Westbrook J, Pawsey M, Greenfield D, Naylor J, ledema R, et al A prospective, multi-method, multi-disciplinary, multi-level, collaborative, social-organisational design for researching health sector accreditation [LP0560737]. BMC Health Serv Res. 2006;6:113.

50. Hinchcliff R, Greenfield D, Moldovan M, Pawsey M, Mumford V, Westbrook J et al. Evaluation of current Australian health service accreditation processes (ACCREDIT-CAP): protocol for a mixed-method research project. BMJ Open. 2012;2(4). doi: 10.1136/bmjopen-2012-001726.

51. Ng K, Leung GK, Johnston JM, Cowling BJ. Factors affecting implementation of accreditation programmes and the impact of the accreditation process on quality improvement in hospitals: a SWOT analysis. Hong Kong Med J. 2013;19(5):434-46.

\section{Submit your next manuscript to BioMed Central and take full advantage of:}

- Convenient online submission

- Thorough peer review

- No space constraints or color figure charges

- Immediate publication on acceptance

- Inclusion in PubMed, CAS, Scopus and Google Scholar

- Research which is freely available for redistribution 